G.A. BARTELDS, J. BRUINING and P. ZITHA

Delft University of Technology, Faculty of Applied Earth Sciences, PO Box 5028, 2600 GA Delft, The Netherlands

\begin{abstract}
An important aspect in polymer flooding is the existence of inaccessible and excluded pore volume in the reservoir. This leads to velocity enhancement i.e. polymers move faster through the porous medium than the water in which they are dissolved. In the conventional description the velocity enhancement factor is taken to be constant.

We derived new model equations based on a percolation model. It turns out that the velocity enhancement factor can be expressed in terms of relative permeabilities and is therefore saturation dependent. This results in an accumulation of polymers at the oil interface. We verified the theoretical findings with two-phase polymer flow experiments. Our experimental results show indeed a considerable pile-up of polymers at the interface.
\end{abstract}

\title{
Introduction
}

Polymer flooding is generally believed to be a well understood process. Yet one of the aspects that is not properly understood is the effect of velocity enhancement. When a polymer solution in a tracered brine is injected into a porous medium polymer molecules break through significantly earlier at the production end than the tracer molecules [1]. The explanation for this velocity enhancement effect is that not all of the pore space is available to the polymer molecules. In a porous medium there exists inaccessible pore volume (IPV) and excluded pore volume (EPV). Inaccessible pores are too small to contain the large polymer molecules, see figure 1. Excluded pore volume is the part of the pore volume with a reduced polymer concentration due to exclusion of the larger polymer molecules from the vicinity of the pore wall. Polymer molecules tend to concentrate in the centre of the pores at the higher velocities of the velocity profile, see figure 2.

The velocity enhancement factor $\alpha$ is defined as the ratio of the average interstitial velocity of the polymer component and the average interstitial velocity of the water phase; $\alpha=\frac{v_{p}}{v_{w}}$. In two-phase flow models of polymer flooding, $\alpha$ is usually assumed to be a constant factor, independent of the saturation level. In these models [2] the velocity enhancement factor is defined as the ratio of the porosity $\phi$ and the effective porosity for the polymer $\phi_{\text {eff }}$. However, the mathematical model for two-phase flow with a constant velocity enhancement factor $\alpha$ is ill posed when dispersive terms are ignored [3]. Ligthelm [4] suggested a more realistic velocity enhancement factor depending on both the water saturation and the relative permeabilities. His results seem very promising although two-phase experimental evidence for velocity enhancement and its possible consequences is still lacking. Kolodziej [5] did preliminary experiments, showing accumulation of polymers at the oil interface, without providing additional explanation.

The objective of this paper is to demonstrate the effect of IPV and EPV on polymer flooding. To this end we present a one-dimensional mathematical model of polymer flooding in which velocity enhancement is included by means of a percolation model [6]. In addition we present laboratory experiments that illustrate the effects of velocity enhancement on polymer flooding. 


\section{Physical model}

The physical model for polymer flooding, includes the following assumptions: (1) The flow is one-dimensional. (2) Both phases are incompressible, and Darcy's Law is valid for both phases. (3) The polymer solutions shows Newtonian behavior, i.e. the velocities are low. (4) Polymer dispersion, gravity and capillary forces are disregarded. (5) The reservoir is homogeneous and water-wet. (6) Adsorption to the porous medium is an increasing function of the polymer concentration.

We have described velocity enhancement with a percolation model. In this model we distinguish three phases: (1) Water which cannot contain polymer (phase 1, with water saturation $S_{w 1}$ ). (2) Water which may potentially contain polymer (phase 2 , with water saturation $S_{w 2}$ ). (3) Oil without polymer (oil phase, with oil saturation $S_{0}$ ). Exchange of water molecules between water phase 1 and 2 is possible. Polymer however is restricted to remain in water phase 2 . The distinction between the two water phases comprises both IPV and EPV, because it does not specify whether phase 1 and 2 belong to the same pore or to different pores.

In the percolation model the effects of IPV and EPV are separated. Figure 3 shows a schematic pore distribution function; $r$ is the radius of the pore/tube, $\psi(r) \mathrm{d} r$ denotes the fractional volume occupied by these pores, $r_{\delta}$ is the maximum radius for which a pore is inaccessible for the large polymer molecules, and $r_{w}$ denotes the radius of pores allowed for water at a certain water moment, during the displacement process. The light grey area denotes the pore space filled with water phase 1, the dark grey area indicates the pore space saturated with water phase 2, and the black area denotes the pore space filled with oil. The water saturation in the medium that is represented by this figure is to such an extend that there exist still isolated clusters of pores with a radius $r<r_{w}$ that are occupied by oil, because the injected water cannot reach them.

The IPV is taken into account by assuming that the smallest pores, with a radius $r$ smaller than a critical radius $r_{\delta}$, do not contain the large polymer molecules. The underlying assumption is that small pores also have small pore throats giving access to those pores. The small pore throats actually prevent that polymer enters the small pores. In water-wet media water prefers to enter the pores in a sequence of increasing radius. This means that all accessible pores with radius $r<r_{\delta}$ must be filled with water before the polymer can enter the porous medium.

The EPV is taken into account by representing each pore as a capillary tube. The large polymer molecule can only flow through the inner part of the tube. There exists a zone near the pore wall that is depleted of polymer molecules. The width of this zone is determined by a characteristic length $\delta$, that is in the order of magnitude of the length of the polymer molecules. In figure 3 fractional volume occupied by EPV is the dark grey area minus the dark grey area consisting of pores with a radius smaller than the critical radius $r_{\delta}$.

\section{Mathematical model}

The main equations of the mathematical model are the conservation equations for oil water and polymer. The conservation equation for the water and the polymer are [2].

$$
\begin{aligned}
& \phi \frac{\partial S_{w}}{\partial t}+u \frac{\partial f_{w}}{\partial x}=0 \\
& \phi \frac{\partial c S_{w}}{\partial t}+\phi \frac{\partial a}{\partial t}+u \frac{\partial \alpha c f_{w}}{\partial x}=0
\end{aligned}
$$

where $\phi$ is the porosity of the medium, $S_{w}$ is the water saturation, $c$ the polymer concentration, uis the total Darcy velocity, $f_{w}\left(S_{w}, c\right)$ is the fractional flow function for water, $a(c)$ is the concentration of polymer adsorbed to the porous medium, and $\alpha$ is the velocity enhancement factor. For our percolation model the velocity enhancement factor $\alpha$ becomes

$$
\alpha=\frac{v_{w 2}}{v_{w}}=\frac{u_{w 2}}{S_{w 2}} \frac{S_{w}}{u_{w}}=\frac{u_{w 2}}{u_{w 1}+u_{w 2}} \frac{S_{w 1}+S_{w 2}}{S_{w 2}}
$$


where $v_{w 2}$ and $v_{w}$ are the average interstitial velocities of water phase 2 and the total water phase respectively, $u_{w 1}$ and $u_{w 2}$ are the Darcy velocities of water phase 1 and 2, and $S_{w 1}$ and $S_{w 2}$ are the saturations for water phase 1 and 2 .

Because $\alpha=\alpha\left(S_{w 1}, S_{w 2} ; u_{w 1}, u_{w 2}\right)$ we need to determine the relative permeabilities for the water without polymers $\left(k_{r w 1}\right)$, and for the water with polymers $\left(k_{r w 2}\right)$. Figure 4 shows the three different possibilities that we can encounter:

- $r<r_{\delta} \quad: q_{w 1}$ is a function of $r$ only; $q_{w 2}=0 ; q_{o}=0$

- $r_{\delta}<r<r_{w}: q_{w 1}$ is a function of $r$, and $\delta ; q_{w 2}$ is a function of $r, \delta$, and $\frac{\mu_{w}}{\mu_{o}} ; q_{o}=0$

- $r_{w}<r \quad: q_{w 1}=0 ; q_{w 2}=0 ; q_{o}$ is a function of $r$ only

where $q_{w 1}, q_{w 2}$, and $q_{o}$ are the volumetric flowrates trough a tube of waterphase 1 and 2 and of the oil phase respectively. The exact functions for these flow rates and their derivations are given in [7].

When the water has displaced the oil from the pores with $r<r_{w}$, than we can define the fraction of pores that are allowed for water phase 1 as

$$
Q_{w}=\int_{0}^{r_{w}} \psi(r) \mathrm{d} r
$$

For the polymer molecules it is not possible to enter that part of the porous medium with $r<r_{\delta}$, therefore we define the fraction of pores that are allowed for water phase 2 as

$$
Q_{w 2}=\int_{0}^{r_{w}} \psi(r) \mathrm{d} r-\int_{0}^{r_{s}} \psi(r) \mathrm{d} r
$$

The fraction of pores that are actually occupied by the two waterphases is of course less than the allowed fraction and depends also on the percolation threshold $Q_{c}$. For $Q_{w} \leq Q_{c}$ no water will flow at all, for $Q_{w} \gg Q_{c}$ all of the allowed fraction will also be occupied, and in between these two extremes we use a power law function [8]. These occupied fractions are shown in figure $3: X_{w}^{O}$ is the light grey plus the dark grey area, $X_{w 2}^{O}$ is the light grey area only, and $X_{o}^{O}$ is the black area.

We use these allowed pore fractions to normalize the pore distribution functions for water phase 1, water phase 2 and the oil. The exact formulas are given in [7]. These normalized pore distribution functions are used to average over all the tube radii $r$, to get the following result for the relative permeabilities of the three phases

$$
\begin{aligned}
& k k_{r w 1}=\frac{\phi S_{w}}{\tau} \frac{\int_{0}^{\infty} q_{w 1} \psi_{w}(r) \mathrm{d} r}{\int_{0}^{\infty} \pi \psi_{w}(r) \mathrm{d} r} \\
& k k_{r w 2}=\frac{\phi S_{w}}{\tau} \frac{\int_{0}^{\infty} q_{w 2} \psi_{w 2}(r) \mathrm{d} r}{\int_{0}^{\infty} \pi \psi_{w}(r) \mathrm{d} r} \\
& k k_{r o}=\frac{\phi S_{o}}{\tau} \frac{\int_{0}^{\infty} q_{o} \psi_{o}(r) \mathrm{d} r}{\int_{0}^{\infty} \pi \psi_{o}(r) \mathrm{d} r}
\end{aligned}
$$

where $\tau$ is the tortuosity factor.

\section{Simulation results}

Figures 5 and 6 show the results of computer simulations for the conventional model of polymer flooding with a constant enhancement factor, with a high numerical dispersion and very low numerical dispersion. The solution consists of two shocks (see [2]). The upstream shock separates the polymer-water region from a region without polymer, the so-called connate water bank. The downstream shock separates a region with movable water from a region with connate water only. Owing to the velocity enhancement, the polymer will 
accumulate at the first shock front. With increasing numerical dispersion the second shock is smeared out as well as the the peak in polymer concentration.

Figure 7 shows the saturation dependence of $\alpha$ for the percolation model. Figure 8 shows the simulation result for the percolation model. Again the solution consists of two shocks, but now the peak in polymer concentration occurs at the second shock front. Figure 9 displays the effluent polymer concentration profile.

\section{Experiments}

To verify these theoretical results we carried out several core flood experiments. We used the following procedure: We pre-adsorbed the core with polymer and then saturated it with oil. Next the polymer solution is injected with a constant flowrate to displace the oil. The produced fluid is collected in small fractions. At the end of the experiment we measure the polymer concentration of the produced fluid with the aid of a spectrophotometer. We did this experiment for different polymer injection concentrations.

The results are shown in figure 10. Clearly there is an significant increase of polymer concentration in the firstly produced sample of water. Comparing figures 9 and 10 , we observe a good qualitative agreement of the simulated and experimental profile.

\section{Conclusions}

- Simulations with the conventional model ( $\alpha$ is constant) show accumulation of polymers at the first shock front.

- Simulations with our percolation model ( $\alpha$ is a function of the water saturation and the relative permeabilities) show that accumulation of polymers occurs at the second shock front.

- The results of the experiments are in qualitative agreement with the simulation results of the percolation model.

\section{References}

[1] K.S. Sorbie, A. Parker, and P.J. Clifford. Experimental and theoretical study of polymer flow in porous media. SPE Reservoir Engineering, pages 281-304, August 1987.

[2] G.A. Pope. The application of fractional flow theory to enhanced oil recovery. Soc. Petroleum Engineers J., 20:191-205, 1980.

[3] G.A. Bartelds, J. Bruining, and J. Molenaar. The modeling of velocity enhancement in polymer flooding. Transport in Porous Media, 26:75-88, 1997.

[4] D.J. Ligthelm. The effect of polymer velocity enhancement by excluded pore volume on the efficiency of polymer flooding. submitted for review by SPE, 1996.

[5] Y.E.J. Kolodziej. Transport mechanisms of xanthan biopolymer solutions in porous media. SPE 18090. In Proc. 63rd Annual Technical Conference and Exhibition of the SPE, Houston, Texas, pages 369-384, October 1988.

[6] A. Heiba, M. Sahimi, L.E. Scriven, and H.T. Davis. Percolation theory of two-phase relative permeability. $S P E$ Reservoir Engineering, pages 123-132, February 1992.

[7] G.A. Bartelds. The Infuence of Inaccessible and Excluded Pore Volume on Polymer Flooding. PhD thesis, Delft University of Technology, Department of Applied Earth Science, Dietz Laboratory, To be published 1998.

[8] P. Bedrikovetsky and J. Bruining. A percolation based upscaling technique for viscous force dominated water flooding in uncorrelated heterogeneous reservoirs. In Proc. 8th Eurapean IOR Symposium in Vienna, Austria, May 1995. 


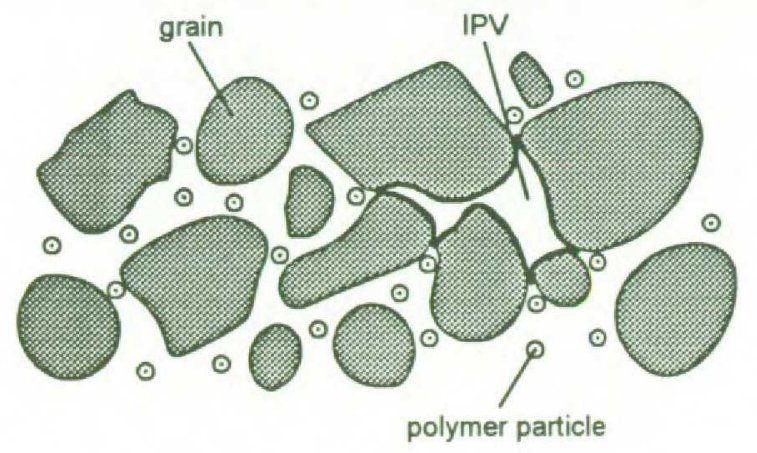

Figure 1: Inaccessible pore volume

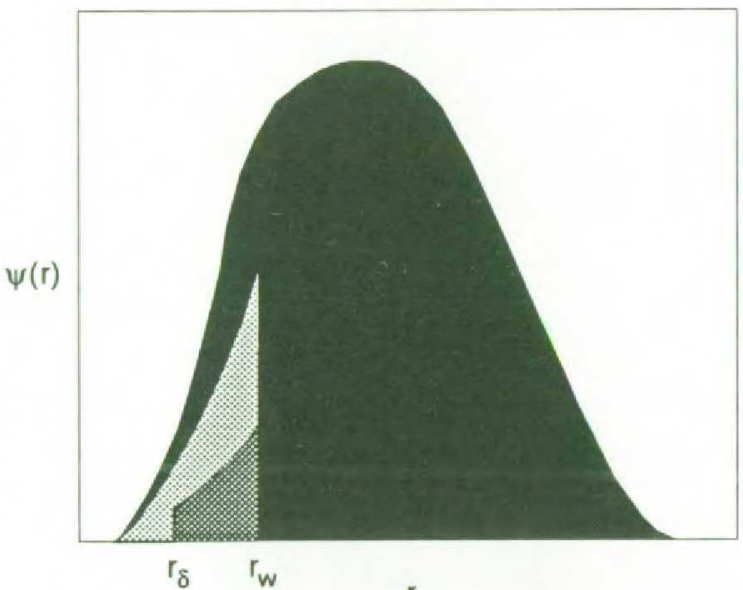

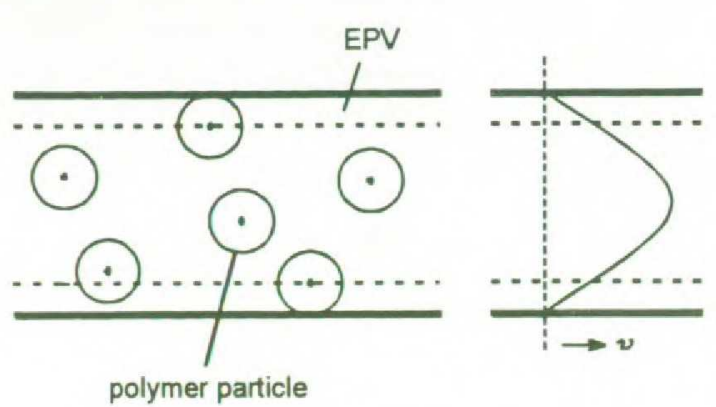

Figure 2: Excluded pore volume

$$
\begin{aligned}
& =x_{w}^{\circ} \begin{array}{l}
\text { occupied porefraction for } \\
\text { water phase 1 }
\end{array} \\
& =x_{w 2}^{\circ} \begin{array}{l}
\text { occupied porefraction for } \\
\text { water phase 2 }
\end{array} \\
& =x_{0}^{\circ} \quad \text { occupied porefraction for oil }
\end{aligned}
$$

Figure 3: Schematic pore distribution function, with the occupied fraction for the three phases, for a certain water saturation where $r=r_{w}$.

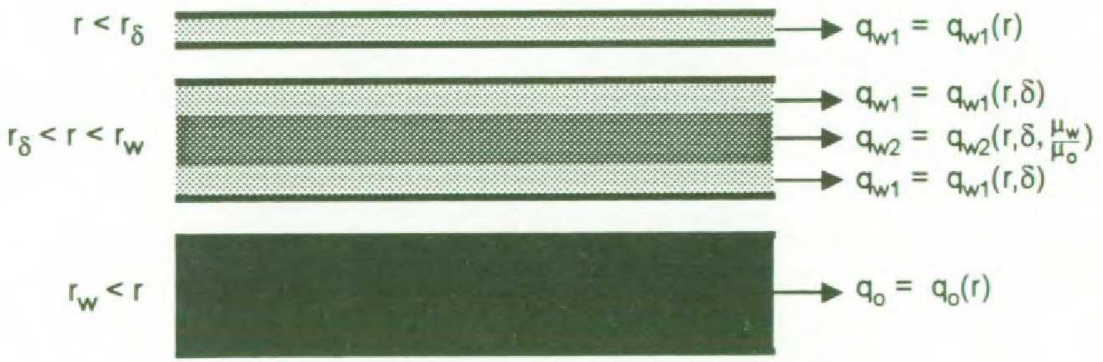

Figure 4: Fluid flow, depending on radius of the tube. 


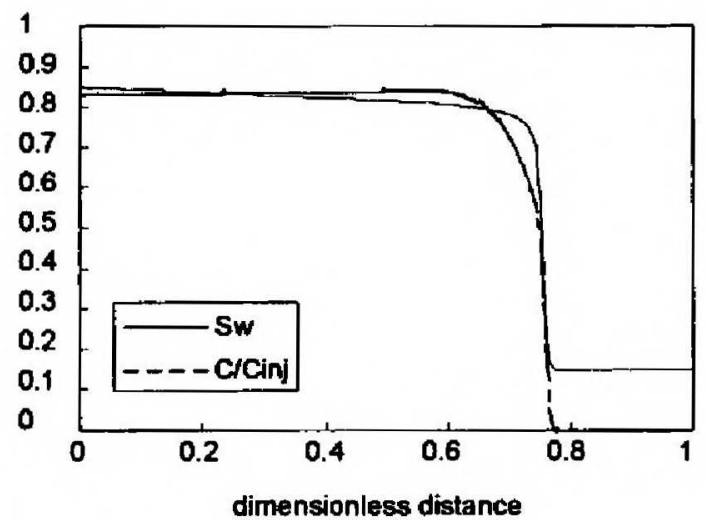

Figure 5: Simulation result for the conventional model with 100 grid blocks.

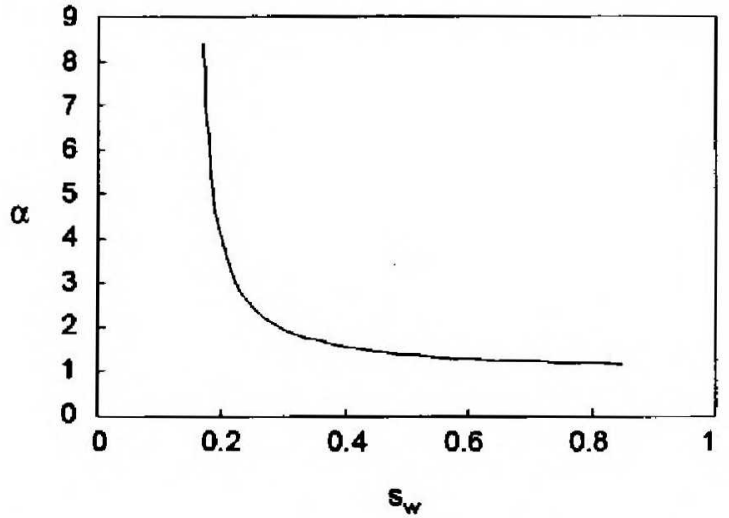

Figure 7: Saturation dependence of $\alpha$ for the percolation model.

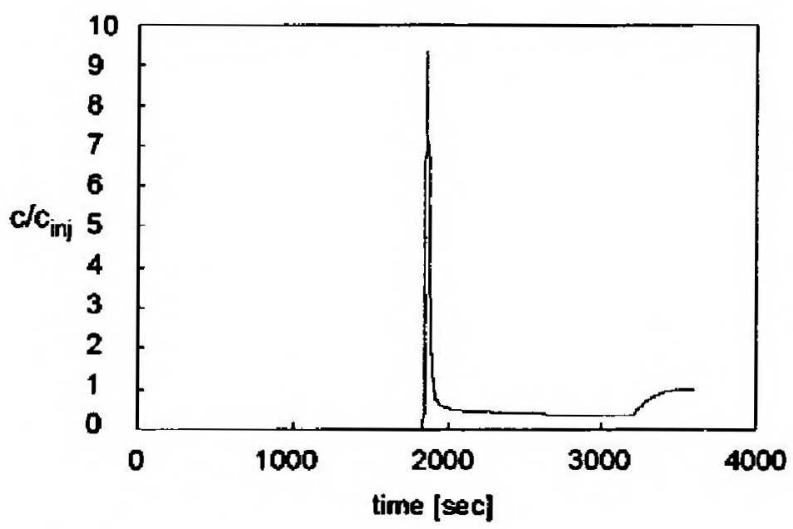

Figure 9: Simulation result for the percolation model (effluent concentration).

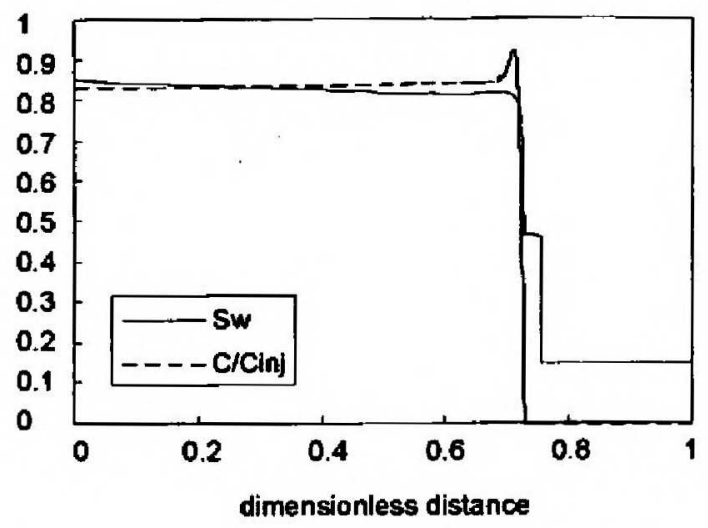

Figure 6: Simulation result for the conventional model with 5000 grid blocks.

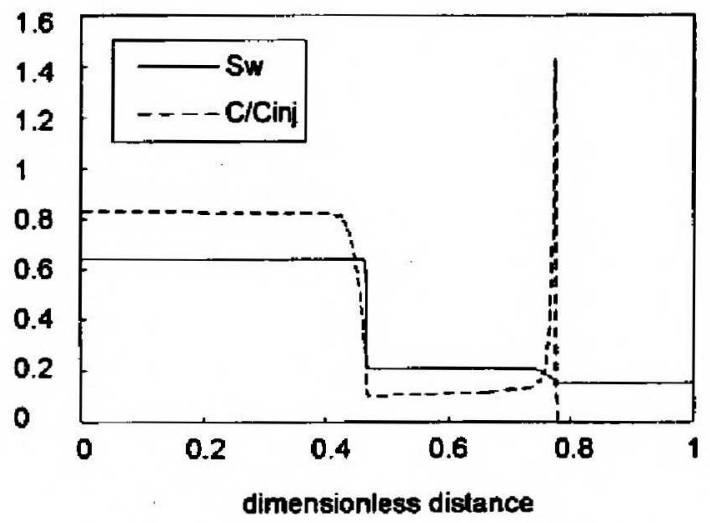

Figure 8: Simulation result for the percolation model (profile).

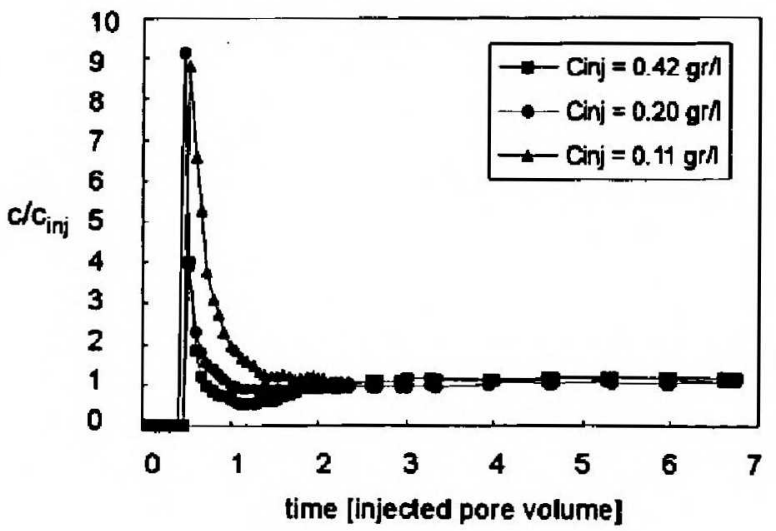

Figure 10: Experimental result: Effluent polymer concentration ratios for different injection concentrations 